\title{
Variabilité journalière de la qualité physico-chimique du lac M'koa de Jacqueville (Côte d'Ivoire)
}

\author{
Yapo Habib KPIDI ${ }^{1 *}$, Ossey Bernard YAPO ${ }^{1,2}$, Tiama Guy BALLET ${ }^{1}$ et \\ Marie-Jeanne OHOU-YAO ${ }^{3}$ \\ ${ }^{1}$ Laboratoire des Sciences de l'Environnement (LSE), UFR des Sciences et Gestion de l'Environnement (UFR- \\ SGE), Université Nangui Abrogoua, 02 BP 801 Abidjan 02 - Côte-d'Ivoire, \\ ${ }^{2}$ Laboratoire Central de l'Environnement, Centre Ivoirien Anti-Pollution (LCE-CIAPOL), Côte-d'Ivoire \\ ${ }^{3}$ UFR Environnement, Université Jean Lorougnon Guédé, BP 150 Daloa, Côte d'Ivoire \\ *Auteur correspondant ; E-mail: kpidiyapoh@gmail.com; Tel: (+225) 78848668
}

\section{RESUME}

La variabilité journalière de la qualité physico-chimique du lac M'koa dépend de facteurs biotiques et abiotiques du cours d'eau qui peuvent interagir entre eux. L'objectif de l'étude est d'évaluer l'influence de ces facteurs sur la variabilité de la qualité physico-chimique par un échantillonnage en continu. Cette variabilité des eaux du lac M'koa de Jacqueville a été étudiée pendant des cycles journaliers de 24 heures en juin et août 2014. Des mesures in-situ et des analyses chimiques portant sur des échantillons prélevés en continu pendant 24 heures sur une station du lac ont été effectuées. Pour chacune des deux périodes d'étude (juin et août), les résultats varient respectivement de 6,07 à 8,08 et de 6,47 à 8,17 pour le $\mathrm{pH}$, de 27,83 à $28,33{ }^{\circ} \mathrm{C}$ et de 26,75 à $28,60{ }^{\circ} \mathrm{C}$ pour la température, de 59,30 à $113,90 \%$ et de 55,95 à $87,34 \%$ pour la saturation en oxygène dissous et de 77,97 à 229,27 et de 23,33 à $176,22 \mathrm{mgO}_{2} / \mathrm{L}$ pour la demande chimique en oxygène. Des variabilités journalières avec des écarts de variation de l'oxygène dissous de 54,60\% et 31,40\% respectivement en juin et août sont notées. L'importance des écarts traduit, en effet, la dépendance des teneurs en oxygène dissous de l'intensité d'une éventuelle prolifération algale et donc caractéristique de l'état trophique avancé du lac M'koa de Jacqueville.

(C) 2017 International Formulae Group. All rights reserved.

Mots clés: Variabilité diurne, nocturne, qualité chimique, oxygène dissous.

\begin{abstract}
The daily variability of the physicochemical quality of M'koa Lake of Jacqueville depends on biotic and abiotic factors of the flowing water that can interact with each other. The objective of the study is to evaluate the influence of these factors on the variability of physicochemical quality by continuous sampling. This variability of M'koa Lake was studied during daily 24-hour cycles on average in june and august 2014. Insitu measurements and chemical analyzes of 24-hours continuous sampling samples of the lake station were conducted. For each of the two study periods, the results varied from 6.07 to 8.08 and 6.47 to 8.17 for the $\mathrm{pH}$, 27.83 to $28.33{ }^{\circ} \mathrm{C}$ and 26.75 to $28.60{ }^{\circ} \mathrm{C}$ for the temperature, from 59.30 to $113.90 \%$ and 55.95 to $87.34 \%$ for dissolved oxygen saturation and from 77.97 to $229.27 \mathrm{mgO}_{2} / \mathrm{L}$ and 23.33 to $176.22 \mathrm{mgO}_{2} / \mathrm{L}$ for the chemical oxygen demand in june and august, respectively. Daily variability with gap of dissolved oxygen of $54.60 \%$ and $31.40 \%$ in june and august are noted, respectively. In fact, the magnitude of these differences reflects the
\end{abstract}


dependence of the dissolved oxygen contents on the intensity of a possible algal proliferation and biomass mineralization and thus characteristic of the degraded physicochemical quality of Jacqueville M'koa Lake. (C) 2017 International Formulae Group. All rights reserved.

Keywords: Diurnal variability, nocturne, chemical quality, dissolved oxygen.

\section{INTRODUCTION}

La problématique de la qualité des eaux de surface constitue l'une des difficultés environnementales majeures à laquelle est confrontée l'humanité (Zalewski, 2002; Fu et al., 2014). La dégradation de la qualité de ces eaux peut provenir des activités agricoles, des effluents industriels et domestiques rejetés dans le milieu récepteur sans traitement préalable. Aussi, la qualité de l'eau se trouve être au cœur de la plupart des problèmes de santé publique que connaissent les pays en développement (Montgomery et Elimelech, 2007; WHO, 2010). Des matières nutritives issues de ces rejets peuvent entraîner une série de changements symptomatiques dont le développement d'algues et de macrophytes qui semblent indésirables et néfastes aux divers usages de l'eau (Mama, 2010). A la base de ces changements, l'oxygène dissous constitue une des variables les plus importantes dans la caractérisation de l'état de santé des systèmes aquatiques (D'Autilia et al., 2004). A l'échelle journalière, les variations d'oxygène dissous, de température et de $\mathrm{pH}$ dans les milieux aquatiques sont fortement dépendantes des activités autotrophes (photosynthèse) et hétérotrophes (eaux usées). L'apport d'éléments nutritifs au milieu favorise l'activité photosynthétique qui influe sur le cycle diurne de l'oxygène dissous. La température et la lumière sont des facteurs environnementaux pré-dominants dans le contrôle de la production primaire
(Sabater et al., 2000). Bien que la variation cyclique de l'oxygène dissous soit bien renseignée, il n'en demeure pas moins que ce processus est peu abordé en écologie aquatique, en qualité de l'eau (Wetzel, 2001). La variabilité diurne de l'oxygène dissous est rarement prise en compte dans les protocoles de suivi de la qualité de l'eau des lacs et réservoirs. La plupart du temps, les concentrations en oxygène dissous, $\mathrm{pH}$ et température sont mesurés de façon ponctuelle pendant le jour. Ces mesures sont faites durant la période où leur teneur semble la plus élevée. Souvent, ces valeurs ne sont pas représentatives des conditions d'oxygénation réelles des cours d'eau (Moatar et al., 2009). Ainsi, Wetzel et Likens (2000) ont montré qu'il est possible de faire plusieurs mesures d'un plan d'eau pour disposer d'une estimation des conditions moyennes d'oxygénation pour une période de temps donnée. Pour obtenir un portrait représentatif de la variabilité de la concentration en oxygène dissous, du $\mathrm{pH}$ et de la température, des mesures devraient être effectuées en continu pendant 24 heures (Villeneuve et al., 2006). Ces mesures permettraient de relativiser les diagnostics établis à partir de mesures ponctuelles et donc de prévenir ou d'anticiper d'éventuels contentieux dans l'évaluation de l'état écologique d'un système aquatique. L'objectif est donc d'évaluer l'influence de certains facteurs sur la 
variabilité journalière de la qualité physicochimique du lac M'koa par un échantillonnage en continu.

\section{MATERIEL ET METHODES}

Présentation de la zone d'étude

Situé dans la région des grands ponts, à $62 \mathrm{~km}$ à l'Ouest d'Abidjan (climat subéquatorial de type Attiéen), le lac M'koa de Jacqueville, lac endoréique sans émissaire de surface constitue le cadre de cette étude (Figure 1). Lac naturel peu profond avec une profondeur maximale de $3,9 \mathrm{~m}$ et une superficie avoisinant $0,180 \mathrm{~km}^{2}$, le lac M'koa est alimenté par infiltration. L'assainissement dans cette localité est de type autonome. La gestion des eaux usées est individuelle.

\section{Recherche de sources de pollution}

Une enquête exploratoire, basée sur des entretiens relativement à la gestion et à l'état du lac, des observations de terrain et des prises de vue, a permis de faire un inventaire des sources potentielles de pollution du lac.

\section{Echantillonnage}

Pour réaliser l'échantillonnage en continu du lac M'koa de Jacqueville pendant 24 heures en moyenne, un échantillonneur automatique Hach Lange $\mathrm{GmbH}$ type $\mathrm{BL}$ 2000 a été posé en un point $\mathrm{A}$ de coordonnées $5^{\circ} 12,415^{\prime} \mathrm{N}-4^{\circ} 25,045^{\prime} \mathrm{W}$ (Figure 2). Ce point a été retenu en raison de son accessibilité et une éventuelle sécurité de la logistique. Le prélèvement en continu a été effectué à $0,5 \mathrm{~m}$ de la surface et a permis d'apprécier l'effet des sources potentielles de pollution sur la variabilité de la qualité de l'eau. Deux campagnes de prélèvement dont la première, du 20 au 23 juin 2014 (grande saison de pluie) et la seconde du 15 au 17 août (saison de crue) 2014 ont été effectuées. La première campagne en continu a permis de collecter 72 échantillons et, la seconde, 48 échantillons. Après prélèvement, ces échantillons ont été mis dans une glacière et conservés à $4{ }^{\circ} \mathrm{C}$ jusqu'au Laboratoire Central de l'Environnement du Centre Ivoirien AntiPollution (LCE-CIAPOL) pour des analyses chimiques.

\section{Analyses}

Le $\mathrm{pH}$ et la température ont été mesurés par le multiparamètre HACH HQ 40d. La mesure de la saturation en oxygène dissous (\%) a été effectuée à l'aide de l'oxymètre HANNA HI 9146. Toutes ces mesures ont été faites in situ (Afnor, 2001; Rodier, 2009). La demande chimique en oxygène (DCO) a été dosée par la méthode de reflux en système fermé suivi d'un dosage par colorimétrie (Ceaeq, 2014) et la lecture est faite au spectrophotomètre UV 2700.

Les moyennes horaires journalières des campagnes de juin et août ont été établies. 


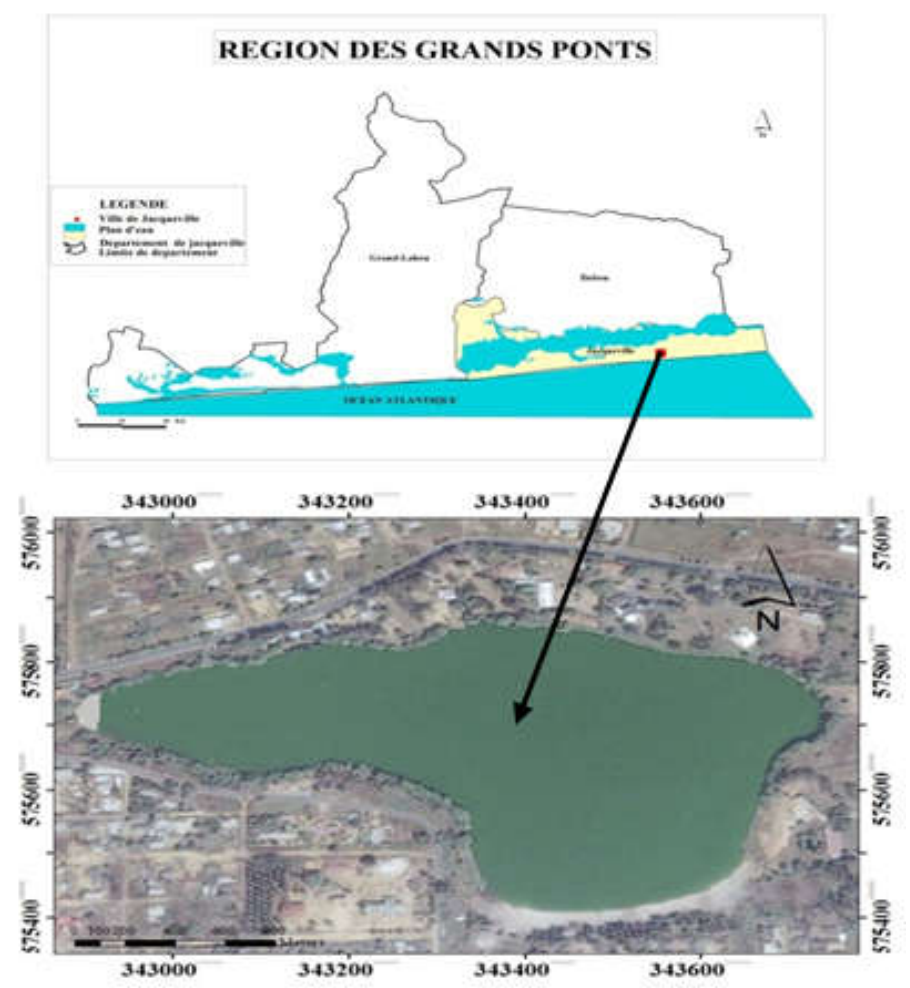

Figure 1: Situation de Jacqueville et de son lac M'koa.

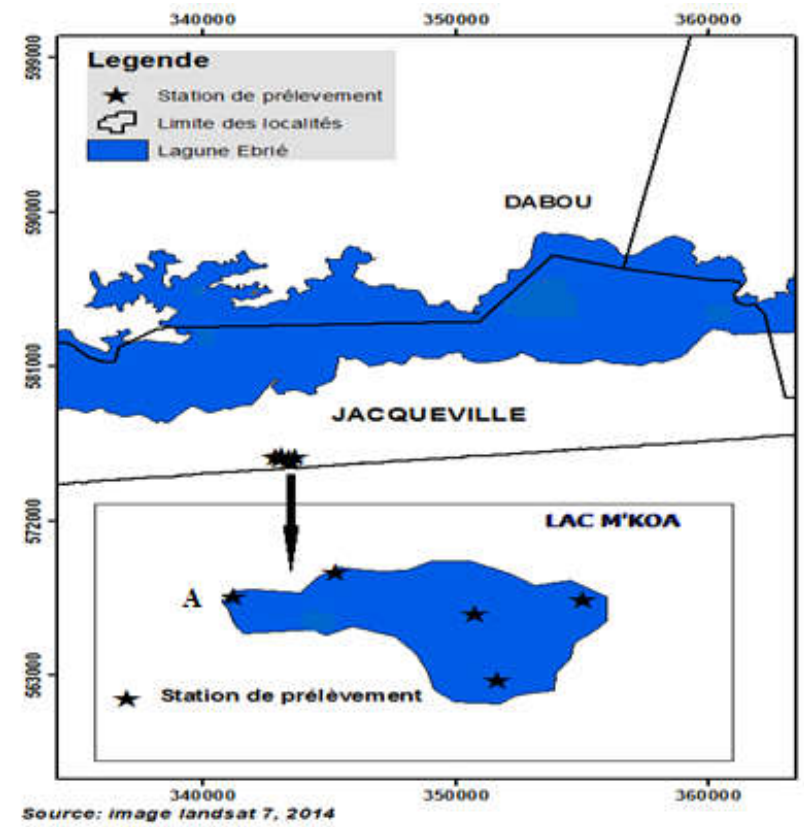

Figure 2: Stations de prélèvement au lac M'koa / Image landsat (2014) modifiée. 
RESULTATS

\section{Inventaire des sources potentielles de pollution au lac}

La Figure 3 présente des raccordements par lesquels transitent les eaux usées rejetées des habitations au lac. Ces rejets peuvent être des sources potentielles d'enrichissement en matières organique, azote et phosphore capables d'influer sur la teneur de saturation en oxygène dissous.

La Figure 4 montre des lavandiers communément appelés "fanico" exerçant leur activité quotidienne au lac. Cette activité est source d'enrichissement des eaux du lac M'koa en phosphore et détergents. Les eaux du lac ont été, aussi, enrichies en matières organiques capables d'utiliser l'oxygène dissous du milieu pour sa dégradation et en nutriments grâce à certaines activités domestiques (vaisselles) qui y sont effectuées. Ces différentes activités sont des sources majeures de pollution des eaux du lac qui peuvent constituer des facteurs de variation de la qualité physico-chimique des eaux du lac M'koa de Jacqueville.

\section{Caractéristiques physico-chimiques de l'eau du lac M'koa}

La variabilité journalière de certains paramètres indicateurs de la qualité des eaux du lac M'koa a été analysée sur deux campagnes dont l'une en juin (grande saison de pluie) et l'autre en août (saison de crue). Leur évolution moyenne horaire sur 24 heures a été relevée.

\section{Variation de pH, température et de la saturation en oxygène au lac M'koa}

La Figure 5 présente les variabilités journalières $\mathrm{du} \mathrm{pH}(\mathrm{A})$ et de la température (B) de juin et août 2014. En juin, une augmentation du $\mathrm{pH}$ allant de $6,11(7 \mathrm{H})$ à 8,08 $(15 \mathrm{H})$ suivie d'une baisse pour atteindre son minimum à $6 \mathrm{H}(6,07)$ avec une moyenne 6,54 ont été constatées. En août, par contre, une augmentation et des pics de décroissance par moment dus à des évènements pluvieux ont été observés. Des écarts de pH de 2.01 et 1.67 respectivement en juin et août ont été notés.

Une augmentation de la température de $7 \mathrm{H}\left(27,83{ }^{\circ} \mathrm{C}\right)$ pour atteindre son maximum à $15 \mathrm{H}\left(28,33^{\circ} \mathrm{C}\right)$ suivie d'une diminution pour une valeur minimale à $6 \mathrm{H}\left(27,83^{\circ} \mathrm{C}\right)$ ont été constatées en juin. En août, les températures minimale et maximale sont relevées respectivement à $6 \mathrm{H}\left(26,75^{\circ} \mathrm{C}\right)$ et $15 \mathrm{H}(28,60$ ${ }^{\circ} \mathrm{C}$ ) avec quelques discontinuités observées à certaines heures suite à des évènements pluvieux-orageux. Des moyennes générales de $28,06{ }^{\circ} \mathrm{C}$ et de $27,63{ }^{\circ} \mathrm{C}$ ont été déterminées respectivement en juin et août 2014. Pour la température, des écarts respectifs de $0,5^{\circ} \mathrm{C}$ et de $1,85^{\circ} \mathrm{C}$ en juin et août ont été enregistrés.

La variabilité journalière de la saturation en oxygène dissous (\%) en juin et août est présentée à la Figure 6.

La saturation en oxygène dissous est croissante, en période diurne de $7 \mathrm{H}(63,07 \%)$ à $15 \mathrm{H}$ où il atteint son maximum de saturation $(113,9 \%)$. Il décroit les heures suivantes, en période nocturne, jusqu'à $6 \mathrm{H}$ où il atteint un minimum de $59,30 \%$ en Juin pour une moyenne de $78,53 \%$. En août, avec les orages et autres perturbations climatiques, une évolution discontinue de l'oxygène dissous du milieu est observée pendant la journée. Durant cette période, la moyenne est $69,91 \%$. Des amplitudes de $54,60 \%$ et $31,40 \%$, respectivement en juin et août, sont constatées

\section{Pollution organique}

La demande chimique en oxygène (DCO) étant un indicateur de la pollution organique de l'eau, sa variation horaire journalière de juin et août 2014 est présentée à la Figure 7. L'évolution de la demande chimique en oxygène des campagnes de juin et août semblent similaires. Aussi, les teneurs de la demande chimique en oxygène de juin sont plus élevées que celles du mois d'août. 


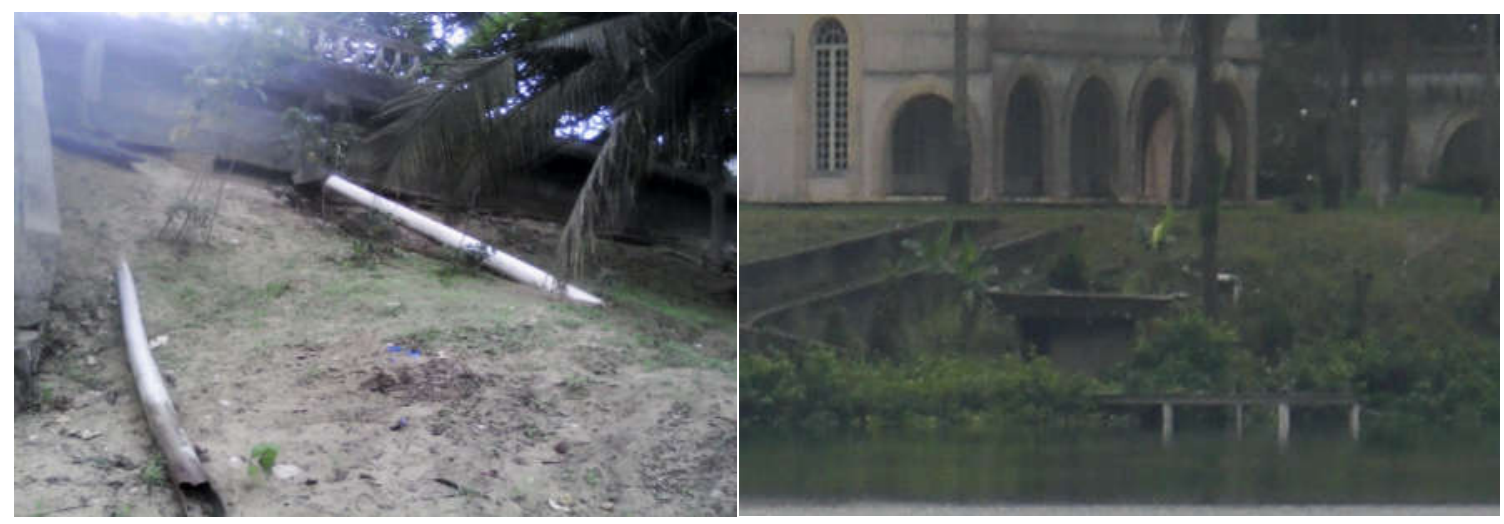

Figure 3: Quelques canaux permettant le transfert des eaux usées domestiques au lac.

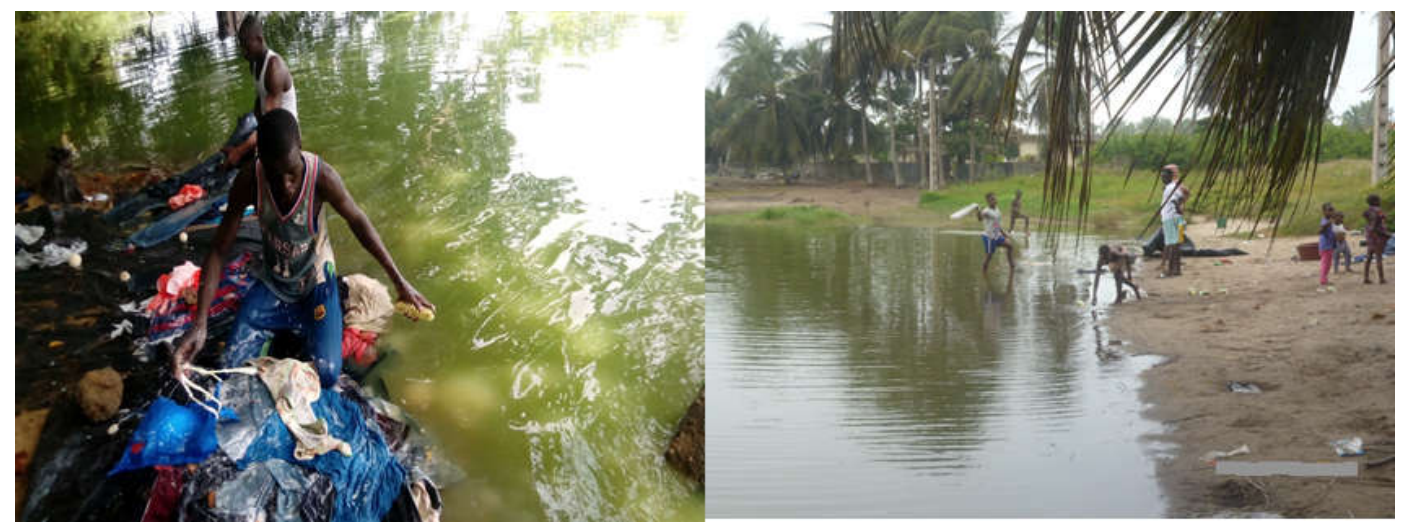

Figure 4: Quelques activités polluantes quotidiennes exercées au lac horaire M'koa.

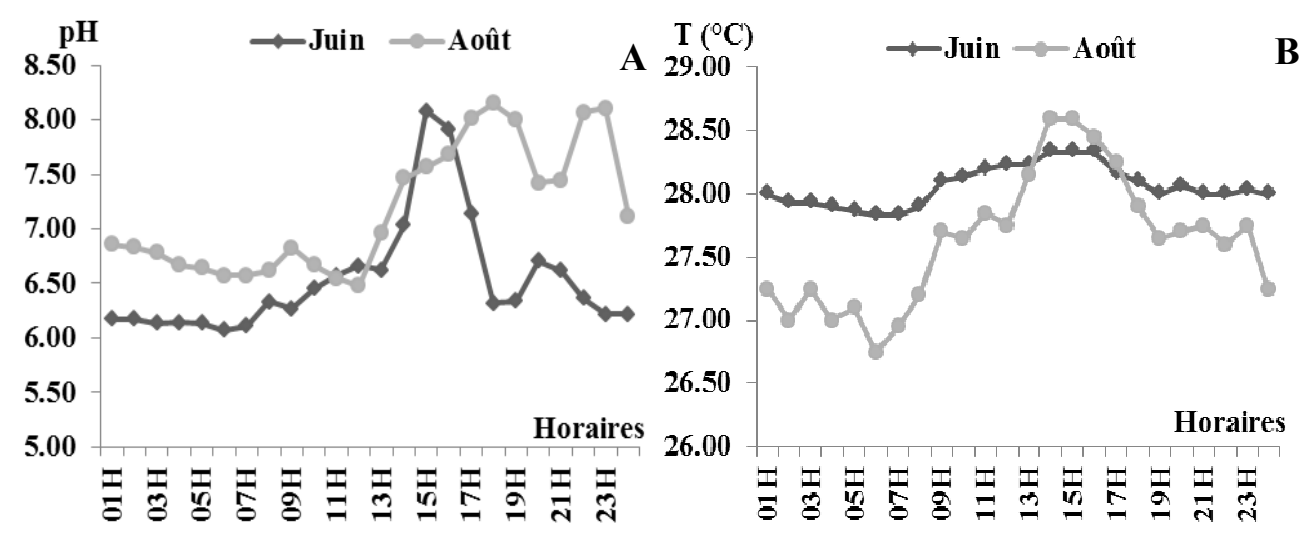

Figure 5: Variation journalière de $\mathrm{pH}(\mathrm{A})$ et de température (B). 


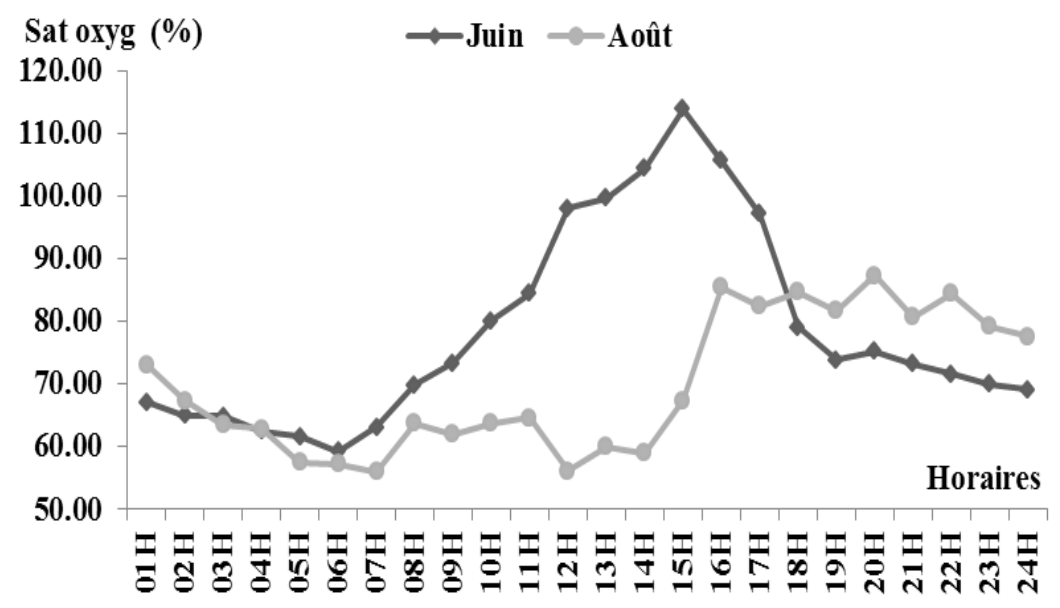

Figure 6: Variation horaire journalière de saturation en oxygène dissous (\%).

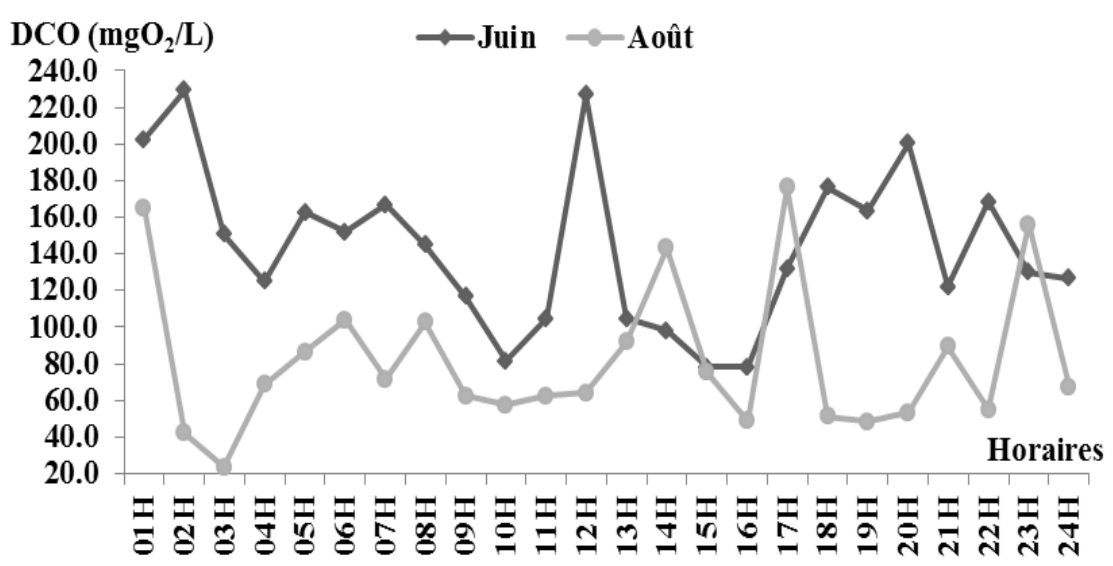

Figure 7: Variation horaire journalière de la demande chimique en oxygène des eaux du lac.

\section{DISCUSSION}

Les eaux usées rejetées dans le lac M'koa par l'intermédiaire des connections illicites des habitations seraient à l'origine de son enrichissement en matières organiques et sels nutritifs (azote et phosphore). Cet enrichissement serait aussi dû aux activités de lavandiers et travaux ménagers effectués au lac. Ces activités pourraient impacter la qualité physico-chimique de l'eau du lac. Ces observations concordent avec celles de N'guessan et al. (2011). Ces auteurs ont montré par des travaux effectués sur les lacs artificiels de Yamoussoukro (Côte-d'Ivoire), que la dégradation de la qualité de ces eaux serait due aux activités anthropiques sur leur bassin versant. Une observation similaire a été faite par Dovonou (2008). Cet auteur a montré que la pollution du lac Nokoué au Bénin, serait liée au mauvais comportement des 
populations riveraines qui jettent dans ce plan d'eau des excrétas. La dégradation de la qualité des eaux pourrait aussi s'expliquer par le lessivage des sols du bassin versant drainant des polluants vers le lac, surtout en période pluvieuse. Ces matières polluantes rejetées dans la colonne d'eau sédimentent dans le lac M'koa peu profond et occasionnent une accumulation de matières organiques et nutritives à l'origine de la dégradation de la qualité du lac M'koa de Jacqueville.

Les matières nutritives, azotes et phosphores en particulier, issues des rejets des eaux usées domestiques et autres activités polluantes, contribueraient à la prolifération des algues phytoplanctoniques dans le lac. En effet, des apports excessifs de nutriments issus des rejets d'eaux usées domestiques, des activités de lavandiers et lessivage du bassin versant du lac M'koa conduiraient à plusieurs types de perturbations des écosystèmes et auraient des conséquences sur les activités humaines: la pêche, la baignade, la potabilisation de l'eau (Durand et al., 2011; Grizzetti et al., 2011). La prolifération des algues phytoplanctoniques serait à l'origine de la coloration verdâtre observée au lac M'koa.

Les résultats ont montré aussi des variations journalières de $\mathrm{pH}$, de température et de saturation en oxygène dissous au lac M'koa. En outre, les valeurs de $\mathrm{pH}$, de température et d'oxygène dissous sont plus élevées en juin qu'en août. Avec un volume d'eau plus important en août, le lessivage des sols a drainé plus de matières organiques et débris importants dans le lac. Ces matières accroîtraient la turbidité de l'eau pour ainsi influer sur la teneur en oxygène dissous par rapport à juin. La solubilité de l'oxygène dans l'eau diminuant lorsque la température augmente, celle-ci induit une baisse de la saturation en oxygène dissous dans l'eau. Cette faiblesse de saturation en oxygène serait surclassée par l'oxygène dissous des activités autotrophes en relation avec la productivité photosynthétique, fonction elle, de la température de l'eau, de la lumière, de la pression atmosphérique, des processus d'oxydation et de décomposition de la matière organique présente dans l'eau. Ainsi, les fortes teneurs en oxygène dissous entre 15 et 16 heures avec une élévation notable du $\mathrm{pH}$ seraient dues à une forte activité photosynthétique dans la couche superficielle de l'eau. Aussi, les écarts d'oxygène dissous et de $\mathrm{pH}$, au sein d'une même journée, seraient caractéristiques des milieux aquatiques très eutrophes (Moatar et al., 2009). Ces résultats sont en accord avec ceux de Sané (2006) et You et al. (2007). Selon ces auteurs, la valeur de $\mathrm{pH}$ serait étroitement liée au niveau d'activité et à l'abondance phytoplanctonique marquée par une coloration verdâtre des eaux du lac. L'oxygène dissous constituerait un paramètre important dans l'évaluation de l'état de santé d'un lac. Il dépend essentiellement de la respiration, de la photosynthèse des populations planctoniques et de la minéralisation de la biomasse (Hebert et Légre, 2000). La photosynthèse et la respiration sont deux processus antagonistes qui régulent le taux de $\mathrm{CO}_{2}$ dans l'eau et dont dépend le $\mathrm{pH}$ d'une eau de surface (Alayat, 2006). En effet, pendant le jour, la photosynthèse entre en jeu et la production d'oxygène dépasse la consommation causée par la respiration de la faune aquatique et la dégradation de la matière organique du milieu aquatique. Il s'en suit une augmentation de la teneur des eaux en oxygène dissous et une consommation de $\mathrm{H}^{+}$favorisant une augmentation du $\mathrm{pH}$ entre $6 \mathrm{H}$ et $15 \mathrm{H}$. Pendant la nuit, la photosynthèse s'arrête, mais les dépenses d'oxygène, déjà signalées, continuent à se manifester (respiration et dégradation de la biomasse). Il s'en suit que le 
bilan de l'oxygène est négatif, et la concentration de ce gaz diminue sensiblement dans les eaux. Aussi, constatons-nous une production de $\mathrm{H}^{+}$à l'origine de la diminution du $\mathrm{pH}$ entre $16 \mathrm{H}$ et $6 \mathrm{H}$.

\section{Conclusion}

La variabilité journalière de la qualité physico-chimique des eaux et, celle de la saturation en oxygène dissous des campagnes de juin et août 2014, en particulier, a été influencée par les activités anthropiques qui demeurent les principales sources d'enrichissement en nutriments et matières organiques du lac, la photosynthèse, la respiration et la minéralisation de la biomasse. Ces facteurs autotrophes et hétérotrophes sont à l'origine de ces variations au lac M'koa.

\section{CONFLITS D'INTERETS}

Aucun conflit d'intérêt n'est à signaler.

\section{CONTRIBUTIONS DES AUTEURS}

YHK a recueilli les résultats au laboratoire. KYH, OBY, TGB et MJO-Y ont guidé et participé à la rédaction de cet article.

\section{REFERENCES}

Alayat H. 2006. Evolution spatio-temporelle du chimisme des eaux thermo minérales des monts de la cheffia (NE algerien). Kartologia, 48: 23-28.

Association française de normalisation (AFNOR). 2001. Qualité de l'eau, Eléments majeurs - autres éléments et composés minéraux. Aubenas Ardèche, $6^{\mathrm{e}}$ Edition, 635p.

Centre d'Expertise en Analyse Environnementale du Québec (CAEQ). 2014. Détermination de la demande chimique en oxygène: Méthode de reflux en système fermé suivi d'un dosage par colorimétrie avec le bichromate de potassium. MA. 315-DCO 1.1, Rév. 3, Ministère du Développement durable, de l'Environnement, de la Faune et des Parcs du Québec, 10p.

D’Autilia R, Falcucci M, Hull V, Parella L. 2004. Short time dissolved oxygen dynamics in shallow water ecosystems. Ecological Modelling, 179: 297-306.

Dovonou F. 2008. La pollution des plans d'eau au Bénin. Diplôme d'Etude Approfondie, Faculté des Lettres Arts et Sciences Humaines, Université d'Abomey-Calavi, Bénin, 67p.

Durand P, Breuer L, Johnes P, Billen G, Butturini A, Pinay G, van Grinsven HJM, Garnier J, Rivett M, Reay DS, Curtis C, Siemens J, Maberly S, Kaste Ø, Humborg C, Loeb R, De Klein J, Hejzlar J, Skoulikidis N, Kortelainen P, Lepistö A, Wright RF. 2011. Nitrogen processes in aquatic ecosystems. In The European Nitrogen Assessment: Sources, Effects and Policy Perspectives, MA Sutton, CM Howard, JW Erisman, G Billen, A Bleeker, P Grennfelt, HJM Van Grinsven, B Grizzetti (Eds). Cambridge University Press: Cambridge; 126 - 146.

Fu F, Dionysiou DD, Liu H. 2014. The use of zero-valent iron for groundwater remediation and wastewater treatment: A review. J. Hazard. Mater, 267: 194- 205.

Grizzetti B, Bouraoui F, Billen G, van Grinsven HJM, Cardoso AC, Thieu V, Garnier J, Curtis C, Howarth RW, Jones P. 2011. Nitrogen as a threat to European water quality. In European Nitrogen Assessment, MA Sutton, CM Howard, JW Erisman, G Billen, A Bleeker, P Grennfelt, HJM Van Grinsven, B Grizzetti (Eds). Cambridge University Press: Cambridge; 379-404.

Hebert S, Légre S. 2000. Direction du suivi de l'état de l'environnement, Ministère de 
l'Environnement Gouvernement $d u$ Québec.

Mama D. 2010. Méthodologie et résultats du diagnostic de l'eutrophisation du lac Nokoué (Benin). Thèse de doctorat, Université de Limoges. 157 p.

Moatar F, Meybeck M, Poirel A. 2009. Variabilité journalière de la qualité des rivières et son incidence sur la surveillance à long terme: exemple de la Loire Moyenne. La Houille Blanche, Revue International de l'Eau, EDP Science, 4: 91-99. DOI:10.1051/1hb/2009050.

Montgomery MA, M Elimelech. 2007. Water and sanitation in developing countries: Including health in the equation. Environmental Science \& Technology, 41(1): 17-24. DOI: 10.1021.

N'Guessan KA, Konan KF, Kotchi YB, Edia OE, Gnagne T, Traore KS, Houenou VP. 2011. Prospects for rehabilitation of manmade lake system of Yamoussoukro (Ivory Coast). Procedia Environmental Sciences, 9: 140-147.

Rodier J, Legube B, Merlet N. 2009. L'analyse de l'Eau. 9è édition. Dunod: Paris. 1579 p.

Sabater S, Armengol J, Comas E, Sabater F, Urrizalqui I, Urrutia I. 2000. Algal biomass in a disturbed Altantic river: water quality relationships and environmental im-plications. Sci. Total Environ., 263: 185-195.
Sane S. 2006. Contrôle de la production primaire du Lac du Guiers au Nord du Sénégal. Thèse de Doctorat de l'Université Cheikh Anta Diop-Dakar, 187p.

Villeneuve V, S Légaré, J Painchaud Warwick V. 2006. Dynamique et modélisation de l'oxygène dissous en rivière. Revue des Sciences de l'Eau, 19(4): 259-274.

Wetzel RG. 2001. Limnology, Lakes and River Ecosystem ( $\left.3^{\mathrm{e}} \mathrm{edn}\right)$. Academic Press, San Diego, 1006p.

Wetzel RG, GE Likens. 2000. Limnogical Analysis $\left(2^{\mathrm{e}}\right.$ edn). Springer-Verlag: NewYork, 429p.

World Health Organization (WHO). 2010. Water, sanitation and hygiene links to health facts and figures-updated. November, 2004, Geneva.

You L, Cui LF, Liu ZW, Yang B, Huan GZF. 2007. Correlation analysis of parameters in algal growth. Environmental Science \& Technology, 30(9): 42-44.

Zalewski M. 2002. Guidelines for the Integrated Management of the Watersheds. Phytotechnoloy and Ecohydrology, Newletter and Technical Publications. Freshwater Management Series, UNEP (5), 237p. 